\title{
A preliminary study of painless and effective transdermal botulinum toxin A delivery by jet nebulization for treatment of primary hyperhidrosis
}

This article was published in the following Dove Press journal:

Drug Design, Development and Therapy

14 July 2014

Number of times this article has been viewed

\author{
Tommaso lannitti ${ }^{1,2}$ \\ Beniamino Palmieri ${ }^{3}$ \\ Anna Aspiro 3 \\ Alessandro Di Cerbo 2,3 \\ 'School of Biomedical Sciences, \\ University of Leeds, Leeds, UK; \\ ${ }^{2}$ Poliambulatorio del Secondo \\ Parere, Modena, Italy; ${ }^{3}$ Department \\ of Surgery and Surgical Specialties, \\ University of Modena and Reggio \\ Emilia Medical School, Surgical \\ Clinic, Modena, Italy
}

\begin{abstract}
Background: Hyperhidrosis is a chronic disease characterized by increased sweat production. Local injections of botulinum toxin A (BTX-A) have been extensively used for treatment of primary hyperhidrosis (idiopathic). The current treatment for this condition involves several intradermal injections, resulting in poor patient compliance due to injection-related pain. Therefore, new protocols, including an improved anesthetic regimen, are required.
\end{abstract}

Aim: We designed the present study to determine whether JetPeel ${ }^{\mathrm{TM}}-3$, a medical device used for transdermal delivery of drugs by jet nebulization, could be used to deliver lidocaine prior to the standard multiple BTX-A injections or deliver lidocaine together with BTX-A in order to determine the protocol giving better results in terms of procedure-related pain, sweating, and patient satisfaction in subjects affected by primary axillary, palmar or plantar hyperhidrosis.

Materials and methods: Twenty patients with a visual analog scale (VAS) sweating score $\geq 8 \mathrm{~cm}$ were randomized to receive lidocaine $2 \%(5 \mathrm{~mL})$ delivered by JetPeel ${ }^{\mathrm{TM}}-3$ followed by multiple injections of BTX-A (100 units) or lidocaine 2\% (5 mL) and BTX-A (50 units) delivered together by JetPeel ${ }^{\mathrm{TM}}-3$. Effect of treatment on sweating was measured by VAS $(0=$ minimum sweating; $10=$ maximum sweating $)$ at 3 -month follow-up. Pain induced by the procedure was assessed by VAS $(0=$ minimum pain; $10=$ maximum pain $)$ immediately after the procedure. Patient satisfaction was assessed at 3-month follow-up using a 5-point scale ( $1=$ not at all satisfied; $2=$ not satisfied; $3=$ partially satisfied; $4=$ satisfied; $5=$ highly satisfied).

Results: Both treatment modalities reduced sweating at 3-month follow-up, if compared with baseline (all $P<0.001$ ). Delivery of lidocaine and BTX-A by JetPeel ${ }^{\text {TM}}-3$ resulted in lower procedurerelated pain and reduced sweating, if compared with lidocaine delivered by JetPeel ${ }^{\mathrm{TM}}-3$ followed by multiple BTX-A injections (all $P<0.001$ ). Patient satisfaction with the procedure was higher in the group receiving lidocaine and BTX-A treatment by JetPeel ${ }^{\mathrm{TM}}-3$, if compared with lidocaine delivered by JetPeel ${ }^{\text {TM}}-3$ followed by multiple BTX-A injections $(P<0.001)$. No side effects were observed in both groups.

Conclusion: Lidocaine and BTX-A can be safely delivered together by JetPeel ${ }^{\mathrm{TM}}-3$ to treat primary palmar, plantar and axillary hyperhidrosis, resulting in lower procedure-related pain, improved sweating and higher patient satisfaction, if compared with lidocaine delivered by JetPeel $^{\mathrm{TM}}-3$ followed by standard BTX-A injection therapy. Our protocol delivering lidocaine and BTX-A together by JetPeel ${ }^{\mathrm{TM}}-3$ requires a reduced quantity of BTX-A, further supporting the use of the transdermal drug delivery by jet nebulization over standard injection therapy for treatment of primary hyperhidrosis.

Keywords: hyperhidrosis, JetPeel ${ }^{\mathrm{TM}}-3$, botulinum toxin A, anesthesia, pain, sweating, transdermal drug delivery, jet nebulization
Correspondence: Tommaso lannitti School of Biomedical Sciences, University of Leeds, Mount Preston Street, Garstang building, Leeds, LS2 9JT, UK

Tel +44 752 I47 I447

Email tommaso.iannitti@gmail.com 


\section{Introduction}

\section{Hyperhidrosis}

Hyperhidrosis is an eccrine sweat gland disorder resulting in an increase in sweating which goes beyond what is physiologically appropriate for temperature regulation, and can affect hands, palms, feet soles, axillae, face, and head. ${ }^{1}$ Primary hyperhidrosis is a disorder of unknown cause (idiopathic) but may be exacerbated by stress. ${ }^{1,2}$ It affects both men and women equally ( $2.8 \%$ of the general population in the United States), ${ }^{3}$ and can be extremely socially debilitating, interfering with work activities and negatively affecting the patients' quality of life., ${ }^{4,5}$

\section{Management of hyperhidrosis}

The management of hyperhidrosis includes the use of topical antiperspirants such as aluminum chloride or tannic acids, oral anticholinergic medications such as glycopyrrolate and propantheline or iontophoresis. ${ }^{6}$ Local injections of botulinum toxin A (BTX-A) are effective in treating primary hyperhidrosis, because BTX-A blocks the release of acetylcholine from the presynaptic nerve terminal with a temporary and reversible local chemodenervation. ${ }^{7-14}$ However, as a great number of painful intradermal injections are required, many patients complain of pain during injections, causing poor compliance in the regular re-injection follow-up. Unfortunately, commonly used pain relief methods such as topical anesthesia, cooling of the skin and use of needles of reduced size ${ }^{15,16}$ have proven unsuccessful, requiring the design of new protocols.

A previous study in our laboratories showed that JetPeel $^{\text {TM}}{ }_{-3}$ (TavTech Ltd., Yehud, Israel) can be used successfully to deliver lidocaine with an anesthetic power superior to topical anesthetic cream in the aesthetic medicine setting. ${ }^{17} \mathrm{In}$ the present study, JetPeel ${ }^{\mathrm{TM}}-3$ was used to deliver lidocaine prior to standard multiple BTX-A injections or to deliver lidocaine plus BTX-A, in order to determine the protocol giving better results in terms of procedure-related pain, sweating, and patient satisfaction.

\section{Materials and methods}

This study was performed in accordance with the Declaration of Helsinki and was approved by the Institutional Review Board at the Poliambulatorio del Secondo Parere (Modena, Italy), where the study was performed.

\section{Patients}

Twenty patients ( 13 women and 7 men) aged $39 \pm 2.63$ years (mean \pm standard error of the mean), were enrolled in this study. All patients signed the informed consent. Only patients affected by primary axillary, palmar or plantar hyperhidrosis with a visual analog scale (VAS) sweating score $\geq 8 \mathrm{~cm}$ participated in this study. Exclusion criteria were neuromuscular disease, concomitant drug treatment interfering with neuroglandular transmission, infections, dermatitis or other skin diseases, allergy or sensitivity to the study medication, previous treatment with BTX-A in the 12 months preceding the study, pregnancy and breastfeeding.

\section{JetPeel ${ }^{\mathrm{TM}}-3$}

JetPeel ${ }^{\mathrm{TM}}-3$ is a medical device which can be used for dermoabrasion, dark spot removal, fine wrinkle smoothing, skin cleansing, squeezing of enlarged pores, dermoepidermal hydration and oxygenation, improvement in microcirculation rheology, dermal lymph drainage and transdermal drug delivery by jet nebulization. Moreover, it can be used to achieve effective skin disinfection and/or sterilization and induce local anesthesia, avoiding the use of needles. ${ }^{17}$ The basic principle of JetPeel ${ }^{\text {TM }}-3$ can be described as follows: a mixture of saline and oxygen is forced into a channel, which accelerates the droplets $(200 \mathrm{~m} / \mathrm{sec})$ outside through a specific nozzle, delivering a powerful jet of microdroplets containing water, drug, air and oxygen (diameter 5-200 $\mu \mathrm{m}$ ) onto the skin surface.

\section{Experimental procedure}

Patients were randomized to receive a single session of 1) lidocaine $2 \%$ (Astra Formedic, Milan, Italy; $5 \mathrm{~mL}$ ) delivered by JetPeel ${ }^{\mathrm{TM}}-3$ and subsequent multiple BTX-A (100 units (U); Bocouture ${ }^{\circledR}$, Merz Aesthetics, Frankfurt am Main, Germany; BTX-A was reconstituted in $5 \mathrm{~mL}$ of saline solution) injections in the area affected by hyperhidrosis (group A [n=10]) or 2) lidocaine $2 \%(5 \mathrm{~mL})$ and BTX-A (50 $\mathrm{U}$ diluted in the lidocaine for each palm, axilla, or foot)
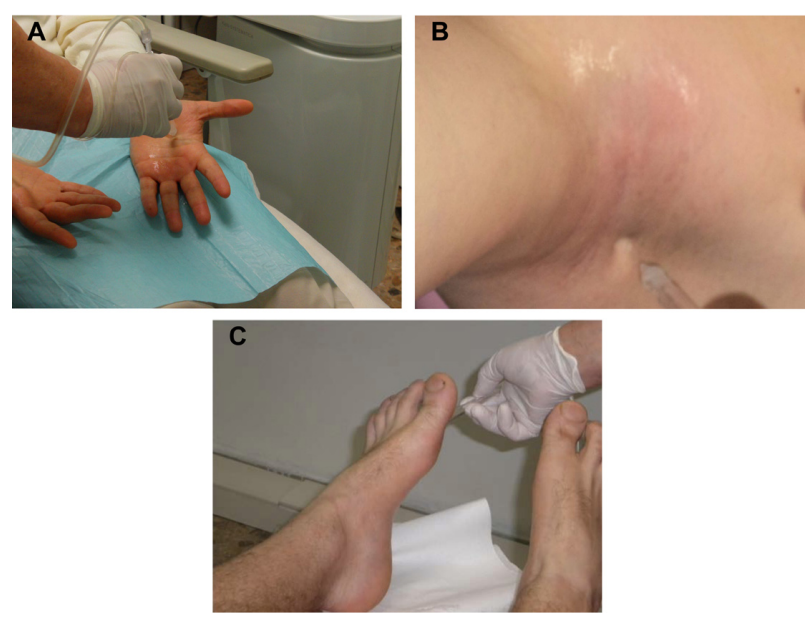

Figure I Treatment of primary palmar (A), axillary (B), and plantar (C) hyperhidrosis with lidocaine and botulinum toxin $A$ delivered by JetPeel ${ }^{\mathrm{TM}}-3$. Note: JetPeel ${ }^{\mathrm{TM}}-3$ is manufactured by TavTech Ltd., Yehud, Israel. 
administered together by JetPeel ${ }^{\mathrm{TM}}$-3 (Figure 1) over the area affected by hyperhidrosis (group $B[n=10]$ ). In patients affected by axillary hyperhidrosis, the area was shaved 2 days before the procedure. Patients were instructed not to use antiperspirants or deodorants for at least 24 hours prior to treatment. Patient follow-up to assess patient satisfaction with the procedure and improvement in sweating was performed at 3 months.

\section{Measurement of procedure-related pain, sweating, and patient satisfaction}

Procedure-related pain was rated by the patients using a $\operatorname{VAS}(0=$ minimum pain; $10=$ maximum pain $)$ immediately

A

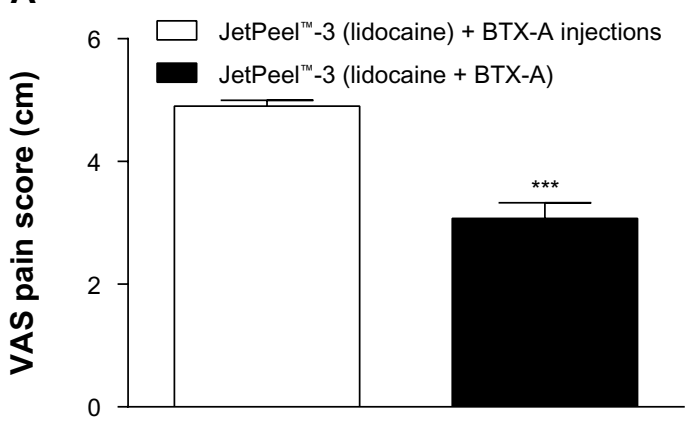

B
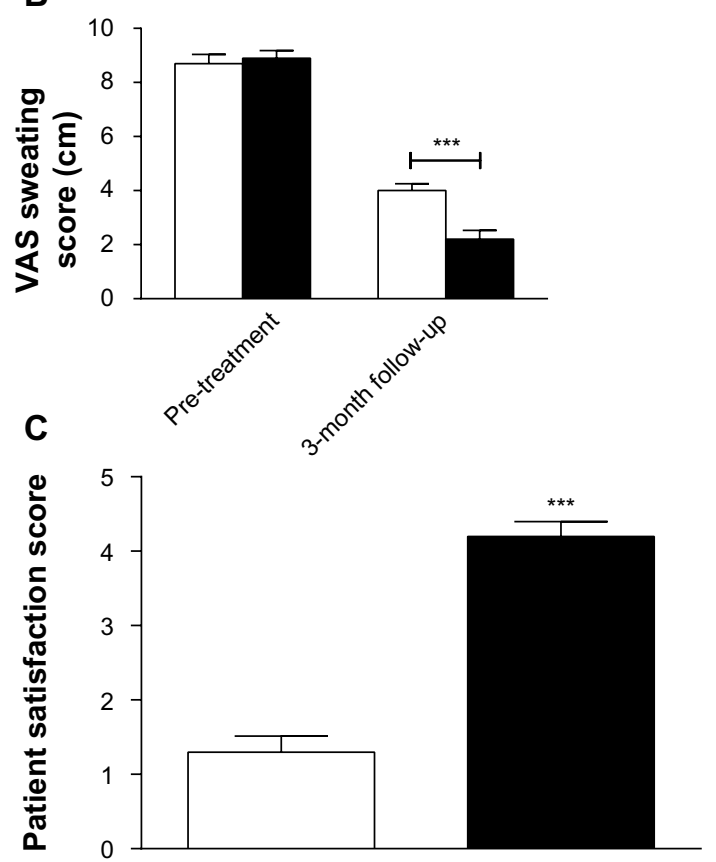

Figure 2 (A) Delivery of lidocaine and BTX-A by JetPeel ${ }^{\mathrm{TM}}-3$ results in lower procedure-related pain versus delivery of lidocaine by JetPeel ${ }^{\mathrm{TM}}-3$ followed by $B T X-A$ injections; (B) delivery of lidocaine and BTX-A by JetPeel ${ }^{\mathrm{TM}}-3$ decreases sweating at 3-month follow-up versus lidocaine delivered by JetPeel ${ }^{\mathrm{TM}}-3$ followed by multiple BTX-A injections; (C) patients are more satisfied with the protocol based on delivery of lidocaine and BTX-A by JetPeel ${ }^{\mathrm{TM}}-3$, if compared with the procedure based on delivery of lidocaine by JetPeel ${ }^{\mathrm{TM}}-3$ followed by BTX-A injections.

Notes: Data are presented as the means \pm standard error of the mean. $* * * P<0.001$. JetPeel ${ }^{\mathrm{TM}}-3$ is manufactured by TavTech Ltd., Yehud, Israel.

Abbreviation: VAS, visual analog scale. after the procedure. The patients were asked to quantify the intensity of sweating using a VAS $(0=$ minimum sweating; $10=$ maximum sweating) before and after treatment. Patients' satisfaction with the procedure was assessed at 3-month follow-up using a 5 -point scale $(1=$ not at all satisfied; $2=$ not satisfied; $3=$ partially satisfied; $4=$ satisfied; $5=$ highly satisfied).

\section{Statistical analysis}

Data were analyzed using GraphPad Prism 6 software (GraphPad Software, Inc., La Jolla, CA, USA). All data are presented as the means \pm standard error of the mean and were first checked for normality using the D'AgostinoPearson normality test. A two-sample unpaired Student's $t$-test was applied to analyze differences in VAS pain score and patient satisfaction score between the two treatment groups. A paired Student's $t$-test was used to compare changes in VAS sweating score at follow-up versus baseline for each treatment modality. Difference in VAS sweating score between the two drug delivery modalities at follow-up was analyzed using a two-way analysis of variance (ANOVA) followed by Sidak's multiple comparisons test.

\section{Results}

In group A, three patients had axillary hyperhidrosis, three had plantar hyperhidrosis and four had palmar hyperhidrosis. In group B, two patients had axillary hyperhidrosis, four had plantar hyperhidrosis and four had palmar hyperhidrosis. Both treatment modalities reduced sweating at 3-month follow-up, if compared with baseline (all $P<0.001$ ). Sweating decreased from a baseline value of $8.7 \pm 0.3 \mathrm{~cm}$ to $4 \pm 0.2 \mathrm{~cm}$ in the group receiving lidocaine by JetPeel ${ }^{\mathrm{TM}}-3$ and multiple BTX-A injections. Sweating decreased from a baseline value of $8.9 \pm 0.2 \mathrm{~cm}$ to $2.2 \pm 0.3 \mathrm{~cm}$ in the group receiving lidocaine and BTX-A by JetPeel ${ }^{\mathrm{TM}_{-}}$. . Delivery of lidocaine and BTX-A by JetPeel ${ }^{\mathrm{TM}}-3$ resulted in lower procedurerelated pain, as assessed immediately after the procedure, and reduced sweating at 3-month follow-up, if compared with lidocaine delivered by JetPeel ${ }^{\mathrm{TM}}-3$ followed by multiple BTX-A injections (all $P<0.001$; Figure 2A and B). Patient satisfaction with the procedure was higher in the group receiving lidocaine and $\mathrm{BTX}-\mathrm{A}$ treatment by JetPeel ${ }^{\mathrm{TM}}-3$, if compared with the group receiving lidocaine by JetPeel ${ }^{\mathrm{TM}}-3$ plus multiple BTX-A injections $(P<0.001$; Figure 2C). Coldrelated pain was observed in a patient affected by axillary hyperhidrosis after BTX-A injections. However, the patient completed the procedure successfully and the pain resolved within 20 minutes without treatment. No side effects were observed in both groups. 


\section{Discussion}

Hyperhidrosis is overproduction of sweat by the exocrine sweat glands and is characterized by enormous psychosocial stress. ${ }^{18,19}$ We found that combined delivery of BTX-A and lidocaine by JetPeel ${ }^{\mathrm{TM}}-3$ resulted in lower procedure-related pain, improved sweating and higher patient satisfaction, if compared with lidocaine delivered by JetPeel ${ }^{\mathrm{TM}}-3$ followed by multiple BTX-A injections into the dermis. The protocol delivering the anesthetic together with BTX-A using JetPeel ${ }^{\mathrm{TM}}-3$ required a reduced quantity of BTX-A, further supporting the use of transdermal drug delivery by jet nebulization over standard injection therapy for treatment of hyperhidrosis. This evidence suggests a more direct penetration of the drug when using JetPeel ${ }^{\mathrm{TM}}-3$. Many studies have investigated the persistence of BTX-A injection efficacy in patients affected by hyperhidrosis. For example, Naver et a ${ }^{20}$ reported a median duration of treatment of 10 months after injecting BTX-A in 170 patients affected by palmar and axillary hyperhidrosis. Furthermore, Schnider et a ${ }^{21}$ injected BTX-A in 13 subjects affected by axillary hyperhidrosis and observed a significant reduction in sweat production over a period of 13 weeks. In a further clinical study ${ }^{22}, X_{\text {Xeomin }}{ }^{\circledR}$, a type of BTX-A, was injected into patients affected by axillary hyperhidrosis, while patients affected by palmar hyperhidrosis, were injected with Xeomin ${ }^{\circledR}$ and Neurobloc ${ }^{\circledR}$, a type of BTX-B. At the 3-week follow-up, all patients, treated for axillary and palmar hyperhidrosis, reported a significant improvement in Dermatology Life Quality Index (DLQI) score. Andrade et a ${ }^{23}$ found two efficient non-invasive methods, iontophoresis and phonophoresis, to administer BTX-A in patients with bilateral primary palmar hyperhidrosis. In this study, BTX-A efficacy lasted over 16 weeks after the end of treatment.

\section{Conclusion}

The present study shows that JetPeel ${ }^{\mathrm{TM}}-3$ can be efficiently and safely used to treat primary palmar, plantar and axillary hyperhidrosis, delivering both anesthetic and BTX-A at the same time. This protocol results in lower procedure-related pain, improved sweating and higher patient satisfaction, if compared with lidocaine delivered by JetPeel ${ }^{\mathrm{TM}}-3$ followed by standard BTX-A injection therapy, also requiring a reduced quantity of BTX-A used. A limitation of our study is the small number of patients involved. Therefore studies in larger cohorts of patients are required.

\section{Acknowledgments}

The authors contributed equally to this work. The authors hereby certify that all work contained in this article is original.
The authors claim full responsibility for the content of the article.

\section{Disclosure}

The authors certify that there is no conflict of interest with any financial organization regarding the material discussed in this article.

\section{References}

1. Naumann M, Dressler D, Hallett M, et al. Evidence-based review and assessment of botulinum neurotoxin for the treatment of secretory disorders. Toxicon. 2013;67:141-152.

2. Atkins JL, Butler PE. Hyperhidrosis: a review of current management. Plast Reconstr Surg. 2002;110(1):222-228.

3. Haider A, Solish N. Focal hyperhidrosis: diagnosis and management. CMAJ. 2005;172(1):69-75.

4. Hornberger J, Grimes K, Naumann M, et al. Recognition, diagnosis, and treatment of primary focal hyperhidrosis. J Am Acad Dermatol. 2004;51(2):274-286.

5. Cohen JL, Cohen G, Solish N, Murray CA. Diagnosis, impact, and management of focal hyperhidrosis: treatment review including botulinum toxin therapy. Facial Plast Surg Clin North Am. 2007;15(1):17-30, v-vi.

6. Scamoni S, Valdatta L, Frigo C, Maggiulli F, Cherubino M. Treatment of primary axillary hyperhidrosis with botulinum toxin type A: our experience in 50 patients from 2007 to 2010. ISRN Dermatol. 2012;2012:702714.

7. Heckmann M, Ceballos-Baumann AO, Plewig G. Botulinum toxin A for axillary hyperhidrosis (excessive sweating). NEngl J Med. 2001;344(7): 488-493.

8. Naumann M, Lowe NJ. Botulinum toxin type A in treatment of bilateral primary axillary hyperhidrosis: randomised, parallel group, double blind, placebo controlled trial. BMJ. 2001;323(7313):596-599.

9. Swartling C, Naver H, Lindberg M. Botulinum A toxin improves life quality in severe primary focal hyperhidrosis. Eur J Neurol. 2001;8(3): 247-252.

10. Salmanpoor R, Rahmanian MJ. Treatment of axillary hyperhidrosis with botulinum-A toxin. Int J Dermatol. 2002;41(7):428-430.

11. Galadari I, Alkaabi J. Botulinum toxin in the treatment of axillary hyperhidrosis. Skinmed. 2003;2(4):209-211.

12. Tan SR, Solish N. Long-term efficacy and quality of life in the treatment of focal hyperhidrosis with botulinum toxin A. Dermatol Surg. 2002;28(6):495-499.

13. Whatling PJ, Collin J. Botulinum toxin injection is an effective treatment for axillary hyperhidrosis. Br J Surg. 2001;88(6):814-815.

14. Heckmann M, Breit S, Ceballos-Baumann A, Schaller M, Plewig G. Side-controlled intradermal injection of botulinum toxin A in recalcitrant axillary hyperhidrosis. J Am Acad Dermatol. 1999;41(6):987-990.

15. Skiveren J, Nordahl Larsen H, Kjaerby E, Larsen R. The influence of needle size on pain perception in patients treated with botulinum toxin A injections for axillary hyperhidrosis. Acta Derm Venereol. 2011;91(1):72-74.

16. Skiveren J, Kjaerby E, Nordahl Larsen H. Cooling by frozen gel pack as pain relief during treatment of axillary hyperhidrosis with botulinum toxin A injections. Acta Derm Venereol. 2008;88(4):366-369.

17. Iannitti T, Capone S, Palmieri B. Short review on face rejuvenation procedures: focus on preoperative antiseptic and anesthetic delivery by JetPeel-3 (a high pressure oxygen delivery device). Minerva Chir. 2011;66(3 Suppl 1):1-8.

18. Solish N, Benohanian A, Kowalski JW. Prospective open-label study of botulinum toxin type $\mathrm{A}$ in patients with axillary hyperhidrosis: effects on functional impairment and quality of life. Dermatol Surg. 2005;31(4):405-413. 
19. Togel B, Greve B, Raulin C. Current therapeutic strategies for hyperhidrosis: a review. Eur J Dermatol. 2002;12(3):219-223.

20. Naver H, Swartling C, Aquilonius SM. Treatment of focal hyperhidrosis with botulinum toxin type A. Brief overview of methodology and 2 years' experience. Eur J Neurol. 1999;6(S4):S117-S120.

21. Schnider P, Binder M, Kittler H, et al. A randomized, double-blind, placebo-controlled trial of botulinum A toxin for severe axillary hyperhidrosis. Br J Dermatol. 1999;140(4):677-680.
22. Rosell K, Hymnelius K, Swartling C. Botulinum toxin type A and B improve quality of life in patients with axillary and palmar hyperhidrosis. Acta Derm Venereol. 2013;93(3):335-339.

23. Andrade PC, Flores GP, Uscello Jde F, Miot HA, Morsoleto MJ. Use of iontophoresis or phonophoresis for delivering on a botulinum toxin $\mathrm{A}$ in the treatment of palmar hyperidrosis: a report on four cases. An Bras Dermatol. 2011;86(6):1243-1246.

\section{Publish your work in this journal}

Drug Design, Development and Therapy is an international, peerreviewed open-access journal that spans the spectrum of drug design and development through to clinical applications. Clinical outcomes, patient safety, and programs for the development and effective, safe, and sustained use of medicines are a feature of the journal, which has also been accepted for indexing on PubMed Central. The manuscript management system is completely online and includes a very quick and fair peer-review system, which is all easy to use. Visit http://www.dovepress.com/testimonials.php to read real quotes from published authors.

Submit your manuscript here: http://www.dovepress.com/drug-design-development-and-therapy-journal 